\title{
A Preliminary Study using PageFetch to Examine the Searching Ability of Children and Adults
}

\author{
James Purvis and Leif Azzopardi \\ Information Retrieval Group, School of Computing Science \\ University of Glasgow, Glasgow, G12 8QQ \\ \{ 0801303P@student, leif@dcs\}.gla.ac.uk
}

\begin{abstract}
Evaluating the children's information seeking behaviors and information retrieval abilities poses a number of difficult challenges for researchers to overcome. One of the main problems is engaging children to undertake search tasks so that their abilities at retrieving relevant information can be assessed. In this poster paper, we outline PageFetch, which is an Information Retrieval based game designed to engage information seekers of all ages, but particular, children, to play and thus provide valuable data to assess and compare their search abilities to other age groups. We also report the results from an initial pilot study using PageFetch where over 140 participants played approximately 1500 games.

While, previous research has shown that children do not perform as well as adults, our finding suggest that given modern search engines, children (or more specifically teenagers) are more than capable of finding specified pages - and in fact for topics that they are more likely to be interested in, they often out perform adults. Since, these findings are very preliminary, they do raise a number of questions about the quality of modern search engines and the search efficacy of younger searchers. This work motivates the undertaking of secondary and larger study that examines on a year by year basis how search skills develop and improve from childhood to adulthood.
\end{abstract}

\section{Categories and Subject Descriptors}

H.5 [Information Interfaces and Presentation]: User Interfaces-Interaction styles; H.3.3 [Information Storage and Retrieval]: Information Search and RetrievalInformation Retrieval

\section{General Terms}

Design, Human Factors

\section{Keywords}

Search, Games, Children, PageHunt, FuFinder

Permission to make digital or hard copies of all or part of this work for personal or classroom use is granted without fee provided that copies are not made or distributed for profit or commercial advantage and that copies bear this notice and the full citation on the first page. To copy otherwise, to republish, to post on servers or to redistribute to lists, requires prior specific permission and/or a fee.

IIiX 2012, Nijmegen, The Netherlands

Copyright 2012 ACM 978-1-4503-1282-0/2012/08 ...\$10.00.

\section{INTRODUCTION}

Children are increasingly using the Web as a source for their education and entertainment, alongside more traditional sources such as printed books and television [8]. However, when using the web it has been reported that children face a number of difficulties and challenges during the course of information seeking and retrieval from querying to browsing to assessing. Firstly, children have difficulty using query-based interfaces. This difficulty has two aspects: there are cognitive barriers in query formulation (not necessarily unique to children); and a lack of typing dexterity results in children having difficulty observing the screen and keyboard at the same time [5]. This often leads to typographical errors and failure to notice events such as suggested queries. Furthermore, children often struggle while searching for information as it requires a developed vocabulary and strong categorisation skills, neither of which are particularly developed in children under the age of 12 [8]. Secondly, children have a lower attention span and often experience difficulty in judging the relevance of a document, which causes them to browse more erratically than adults. This leads to frequent revisiting of previous pages or 'looping', as well as spending less time reading a page [2]. Finally, it has been shown that children prefer a personalised interface that reflects their personality and interests $[1,7,9]$, thus creating a more engaging experience. In this work, we are particularly interested in the first major challenge regarding querying, where we would like to investigate the differences between adults and children in terms of their search ability (or Search-Fu [11]) when it comes to finding webpages. However, to undertake such a study another challenge needs to be overcome. In a study conducted by Druin et al [3], it was found that many children are often uninterested in searching for information online or are only interested in searching for information that is relevant to their personal interests in an experimental setting. Consequently, children who were unmotivated were the least successful in completing information retrieval tasks given by the study. It was suggested that a more effective means of engaging child participants in search studies must be developed in order to gain further insights into the searching behaviours of children. To this end we have developed a game called PageFetch which aims to engage children (aged 8 to 80) in completing named-page search tasks through a fun and interactive game.

In this paper, we shall first discuss and describe some of the recent and most related Human Computational IR games that have been developed, before describing the PageFetch game in Section 2. Then in Section 3, we shall describe 
the pilot study that we undertook before presenting our preliminary results and findings in Section 4. In Section 5 we reflect upon the pilot study and discuss how we intent to improve this initial version of the game to make it not only more appealing and enjoyable, but a better experimental apparatus for conducting such studies.

\section{IR GAMES AND PAGEFETCH}

Usually the primary focus of evaluation is to determine the quality of a retrieval system. However, the performance of users and their ability to use such systems is particularly important as the abilities of different users groups may vary widely. As previously mentioned, it has been reported that children face many difficulties when searching, and often find formal experimental settings daunting. However, with the advent of human computation games [6] it may be possible to couch Information Retrieval tasks within an engaging experience in order to assess the abilities of children (and adults). However, such games provide controlled setting, much like a traditional experiment, in which large amounts of useful and reliable data can be generated at a very low cost. Already a number of games related to PageFetch have been developed within the context of IR and include: (i) Book Explorer [4], (ii) PageHunt [10] and (iii) Fu-Finder [11].

Figure 1 shows a screenshot of the PageFetch ${ }^{1}$. The childfriendly user interface incorporates bright colors and a helpful puppy avatar to help players undertake the search tasks. PageFetch is most similar to PageHunt [10] and Fu-Finder [11], which have been used to improve search engines and study querying behavior, respectively. In each of these games, players are presented in a web page, and they need to enter in a query that retrieves the page. Players have three minutes to try and find as many of the pages as possible. PageFetch is novel, in a number of respects:

1. PageHunt and Fu-Finder used a similar set of pages (a mixture of hard and easy to find pages given Bing's search engine at the time), in PageFetch pages from six different categories were used (cartoons, music, sports, politics) to provide a range of genres for children and adults to find ${ }^{2}$.

2. Thus, players are given a choice of category from which pages are presented (rather than the random pages presented in the other games). The choice of categories provides more focused game play and lets players pick an genre that is of interest to them.

3. Unlike PageHunt, PageFetch assigns points according to how high the results page is returned (i.e. the higher the rank the more points), and PageFetch one retrieves pages using one engine (Bing), as opposed to the three engines used in Fu-Finder.

4. Finally, PageFetch was designed to encourage players to focus on entering successful queries, which is achieved by providing a "no pass" bonus which increases progressively when the player successfully finds a query that returns the page in the top 8 results.

\footnotetext{
${ }_{1}$ The game is accessible from: http://www.dcs.gla.ac.uk/access/ pagefetch.

${ }^{2}$ Pages were selected using trending search terms for under-12s, under-16 and adults from 2011.
}

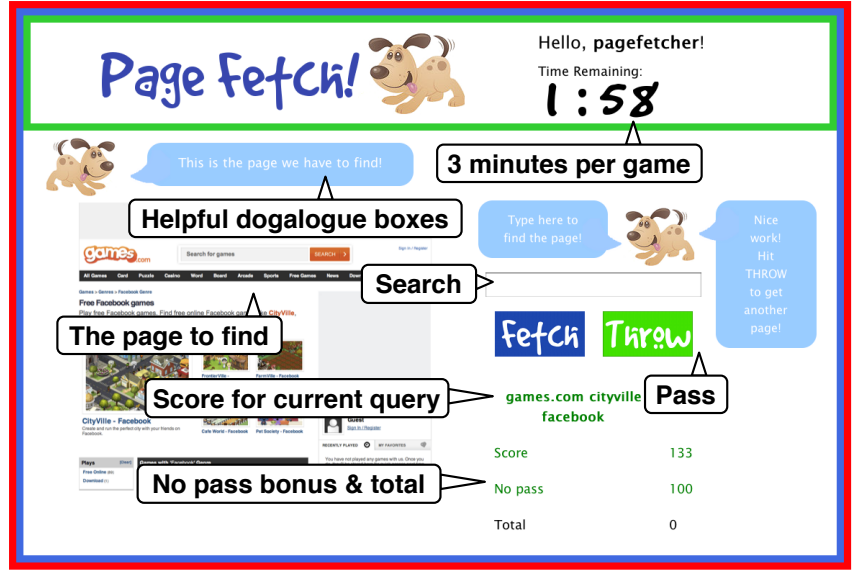

Figure 1: In game PageFetch screenshot.

This tries to stimulate the player into entering reformulations so that they can obtain higher bonuses later on.

This final feature was also included to see whether we could encourage players to improve their searching abilities i.e. helping them to learn to search better. It is envisaged that through repeated gameplay that children (as well as adults) would be able to improve their performance by adopting more successful search strategies.

\section{PILOT STUDY}

The pilot study of the PageFetch game was undertaken from the 24th of February 2012 until the 20th of March, 2012. This study was conducted to test the PageFetch game itself (i.e. find out what works and what does not in terms of game play and layout), and to obtain some preliminary data to provide an indicative of the differences between children and adult searchers. The participants were recruited from a local high school and within the university through flyers advertising the game, and demonstrations of the game at the school and university open days ${ }^{3}$. To encourage participants to play the game we offered iPod Shuffles as prizes to the highest scoring players.

During the four week pilot a total of 142 participants played 1475 games of PageFetch and 8672 queries were issued of which 1653 were unique. An additional 30 participants registered but these users did not complete a full game and so were excluded from our analysis. When considering the results of each experiment, users were divided into three age brackets - under-12s, 12 - 16 year olds, and over-16s. These brackets were based on previous groupings used in children's information retrieval studies $[3,5]$. Table 4 provides a summary of the usage statistics for the different age groups.

\section{RESULTS AND ANALYSIS}

The children in the under-12 age bracket played a considerable number of games despite their small representation and produced 1256 queries. Each player in this group played around 14 games on average. In the 12-16 year olds group, participants played around 10 games on average and

\footnotetext{
${ }^{3}$ Ethics approval was obtained by from our university to conduct this study which included obtaining permission from the local school to contact their students. (ETHICS-CSE00927).
} 


\begin{tabular}{|c|c|c|c|c|c|c|c|}
\hline & $\begin{array}{c}\text { Tot. } \\
\text { \#Players }\end{array}$ & $\begin{array}{c}\text { Tot. } \\
\text { \#Games } \\
\text { Played }\end{array}$ & $\begin{array}{c}\text { Avg. } \\
\text { \#Games } \\
\text { Per Player }\end{array}$ & $\begin{array}{c}\text { Tot. } \\
\text { \#Queries } \\
\text { submitted }\end{array}$ & $\begin{array}{c}\text { Avg. } \\
\text { \#Queries } \\
\text { Per Game }\end{array}$ & $\begin{array}{c}\text { Avg. } \\
\text { \#Chars. } \\
\text { Per Query }\end{array}$ & $\begin{array}{c}\text { Avg. } \\
\text { \#Word } \\
\text { Per Query }\end{array}$ \\
\hline Under-12s & 13 & 186 & 14.31 & 1256 & 6.75 & 32.66 & 5.23 \\
\hline $\mathbf{1 2 - 1 6}$ & 44 & 445 & 10.11 & 2296 & 5.16 & 26.45 & 4.72 \\
\hline Over-16s & 85 & 851 & 10.01 & 5120 & 6.01 & 20.2 & 3.44 \\
\hline
\end{tabular}

Table 1: Usage statistics for each age group.

produced 2296 queries, producing more queries per game than under-12s. Over-16s also played around 10 games on average, and produced more queries per game than under16s. These usage statistics suggest that adults are capable of forming search queries much more quickly than children - this is consistent with previous findings [3]. Also, younger children were inclined to play more games of PageFetch each than adults were suggesting that the incentive or the design of the game was appealing.

When we examined the query logs produced from the game play, we found that the queries posed for certain pages tended to be very similar (or the same). For example, of the 32 queries for the gamepage 'Wikipedia - Pokemon'4, 25 were 'wikipedia pokemon'. The seven other queries expressed were by generated by under-16s - no adult searcher used any query for the page other than 'wikipedia pokemon'. On other pages, this trend was similar, suggesting that adult searchers could more readily identify the salient terms on a page - which led to a greater consistency and less variation in the queries expressed for a page. However, for children searchers the variations were often due to the inclusion of stop words, use of natural language or misspellings. Regardless of these misspellings though the performance of their searches was not adversely affected - as the Bing Search API corrects misspellings in the majority of cases.

We also examined the logs to see whether players were including navigational cues (i.e. partially or fully formed URLs) within the query. Of the submitted queries, only a few were purely URLs (4.3\%) such as 'www.tesco.com', most queries $(87.9 \%)$ contained no navigational cues, and the remainder contained partially formed URLs $(7.8 \%)$ such as 'justjared.com' or 'justjared'.

\subsection{Page and Player Findability}

To examine who easily players could find pages, and how good players were at finding pages, we employed findability measures used in [10] and [11]. Page Findability is defined as the percentage of times the page was successfully retrieved in the top $n$ ranks. While, Player Findability is defined as the percentage of times the player successfully retrieved the given page in the top $n$ ranks. For each analysis we used a $n$ of 8 (as Bing's Search API returns 8 results in the first instance).

Figure 2 displays a histogram of Page Findability Scores. The first notable finding is that 165 pages were never found and attracted a findability score of zero. There are three reasons for this: (i) some pages were not presented during the games played, (ii) some pages rendered poorly and so could not be found, and (iii) some pages were presented and rendered properly but were actually never found. Further analysis is required to determine the breakdowns. However, for the pages presented and found at least once, we can see that the Page Findability is more or less uniform, though the

\footnotetext{
$\overline{4_{\text {http://en.wikipedia.org/wiki/Pokemon }}}$
}

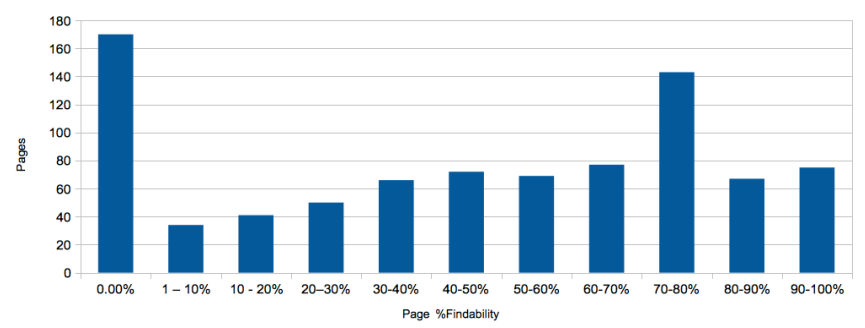

Figure 2: Distribution of Page Findability Scores.

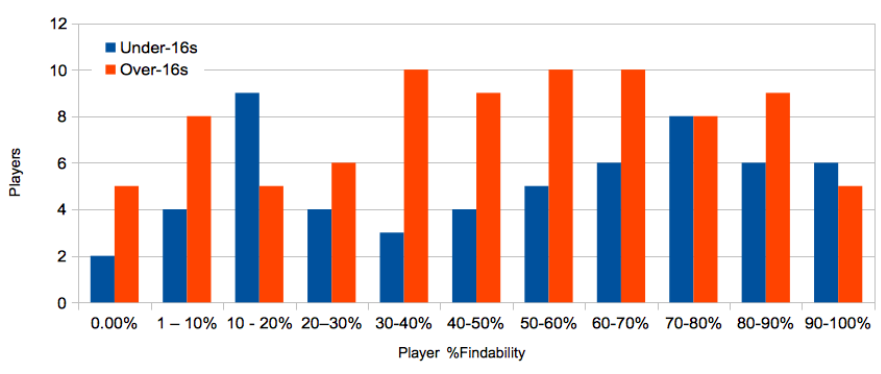

Figure 3: Distribution of Player Findability for under-16 (blue) and over the age of 16 (red).

tendency is that the pages selected tend to be more findable. The mode of the distribution (excluding zero) is at $70-80 \%$ suggesting that most pages, when queried for were found 7-8 times out of 10 query attempts.

Figure 3, on the other hand, displays the histogram of Player Findability Scores, where we grouped the Under-12 with $12-16$ 's as there were only 13 Under-12s. In the over16's group, most players obtained a Player Findability of $30-70 \%$ indicating that they were reasonably successful most of the time. In the under-16's group, there appears to be a bi-modal distribution, where a group of players are centered around $10-20 \%$ and another group of players are centered around $70-80 \%$ Findability. This is an interesting observation, which needs further examination, but it seems to suggest that our child participants (mostly 12-16, or teenagers) are quite adept at searching. However, there is also another group which have not become very proficient at search. However, this may also persist into adulthood as even in the older age group there are still many players that are in the lower findability brackets. Obviously, a larger sample of players is needed before we can make any meaningful comparisons, but perhaps in this age range the differences between adults and teenagers is not so different.

\subsection{Categories}

Game pages within PageFetch were divided into a number of different categories - Sports, Games, Politics, Actors, News and Music. Table 4.2 shows how many times games in each category were played for each demographic. Games, 


\begin{tabular}{|c|c|c|c|c|c|c|}
\hline & 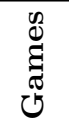 & 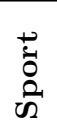 & 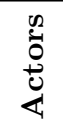 & $\sum^{\infty}$ & 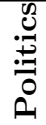 & 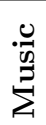 \\
\hline Under-12s & 42 & 89 & 127 & 8 & 3 & 17 \\
\hline $12-16$ & 170 & 97 & 145 & 11 & 17 & 16 \\
\hline Over-16s & 190 & 206 & 131 & 37 & 74 & 40 \\
\hline Total & 402 & 392 & 403 & 56 & 94 & 73 \\
\hline
\end{tabular}

Table 2: The number of games played in each category in each age group.

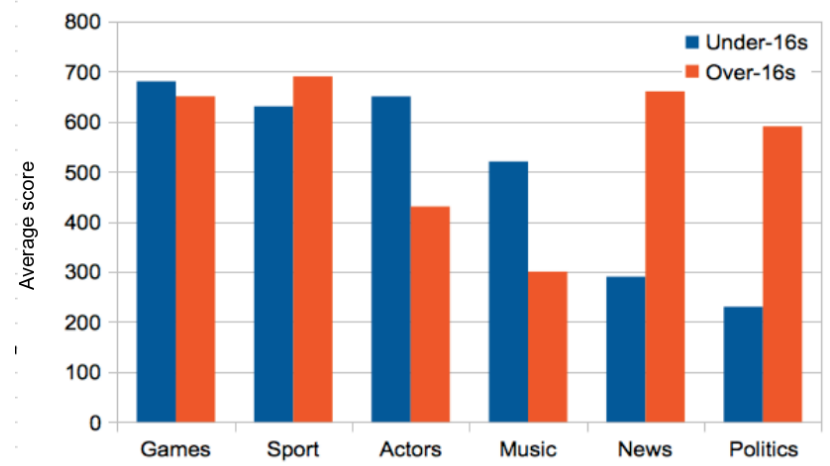

Figure 4: Comparison of performance in each category between over-16s (red) and under-16s (blue).

Sport and Actors were considerably more popular than the other categories. However, Games, Sport and Actors were also the three categories on the top row of the category selection page, suggesting that players were more likely to select the initial categories before choosing the ones further down. In subsequent designs of the game, it may be worth shuffling or rotating the order of the categories on the selection page in order to encourage players to play multiple categories (and to be more in line with a standard experiment).

Figure 4 shows the average performance (y-axis) for each of the different categories for Under-16s and Over-16s. The figure shows that for topics such as news and politics Over16 's markedly outperformed the Under-16s, while on the actors and music categories this trend was reversed. This relates back to the research regarding the motivation of searchers and finding content that is of interest. Here, the high concentration of child-relevant pages were in actors, music and games, where the other categories were not particularly targeted to a younger demographic. These findings suggest that when motivated, and the content is on topic, that they can successfully find it. And similarly for older searchers, where it seems that motivation may also play a part in determining how successful they are too. Further research will be required to explore this in detail.

\section{SUMMARY AND FUTURE WORK}

In this paper, we have described PageFetch, an IR game for children and adults, along with the findings from an initial study using the game. The initial study has provided us with a number of insights on how to improve and refine the game, as well as provided us with a number of interesting directions to examine in further work. Specifically, we plan to conduct a secondary and larger study, where we can examine the performance of searchers from the age of 8 and upwards. This would enable us to more precisely show how the performance of searchers evolves as children develop through their teenage years and into adults. It will also be of interest to determine whether and what differences now exist, in terms of performance, given modern day search engines, since they are very robust to common problems that children face (i.e. misspellings and other query formulation problems.)

Acknowledgements: This research is funded by the European Community's Seventh Framework Programme FP7/ 2007-2013 under grant agreement no. 231507. We would also like to thank Doug Dowie, Richard Glassey and Carly O'Neil for their help and assistance.

\section{REFERENCES}

[1] D. Bilal. Draw and tell: Children as designers of web interfaces. Proceedings of the American Society for Information Science and Technology, 40(1):135-141, 2003.

[2] D. Bilal and J. Kirby. Differences and similarities in information seeking: children and adults as Web users. Information Processing $\&$ Management, 38(5):649-670, 2002.

[3] A. Druin, E. Foss, H. Hutchinson, E. Golub, and L. Hatley. Children's roles using keyword search interfaces at home. In Proceedings of the 28th SIGCHI, pages 413-422, 2010.

[4] G. Kazai, N. Milic-Frayling, and J. Costello,. Towards Methods for the Collective Gathering and Quality Control of Relevance Assessments. In Proceedings of the 32nd International ACM SIGIR Conference on Research and Development in Information Retrieval, SIGIR'09, pages 452-459, Boston, MA, USA, July 2009. ACM.

[5] H. Jochmann-Mannak, T. Huibers, and T. Sanders. Children's information retrieval: beyond examining search strategies and interfaces. In The 2nd BCS-IRSG Symposium: Future Directions in Information Access, eWic Series, pages 64-72, London, September 2008. British Computer Society.

[6] L. von Ahn and L. Dabbish. Designing Games With a Purpose. Communications of the ACM, 51:58-67, August 2008.

[7] A. Large, J. Beheshti, and T. Rahman. Design criteria for childrenâĂŹs web portals: The users speak out. Journal of the American Society for Information Science and Technology, 53:79-94, 2002.

[8] A. Large, V. Nesset, and J. Beheshti. Children as information seekers: what researchers tell us. New Review of Children's Literature and Librarianship, 14(2):121-140, 2008.

[9] J. A. Large and J. Beheshti. Interface design, web portals, and children. Library Trends, 54(2):318-342, 2005.

[10] H. Ma, R. Chandrasekar, C. Quirk, and A. Gupta. Improving search engines using human computation games. In Proceeding of the 18th CIKM, pages 275-284, 2009.

[11] C. O'Neil, J. Purvis, and L. Azzopardi. Fu-finder: a game for studying querying behaviours. In Proceedings of the 20th ACM CIKM, pages 2561-2564, 2011. 


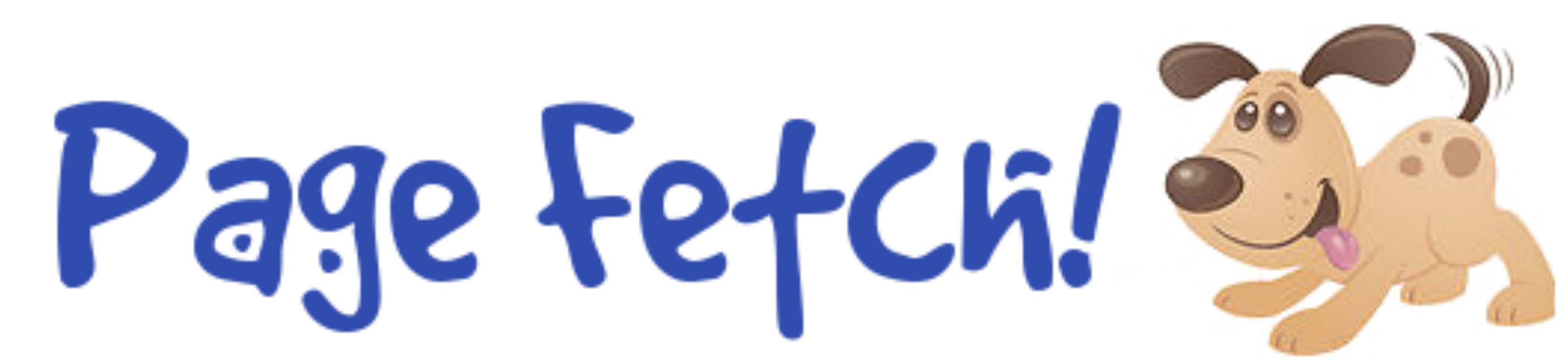

EXAMINING THE SEARCHING ABILITY OF CHILDREN AND ADULTS

\section{James Purvis Leif Azzopardi}

\section{INTRODUCTION}

Children are increasingly using the Web for their education and entertainment, alongside more traditional sources such as books and TV. However, children face a number of challenges:

- cognitive barriers in query formulation

- a lack of typing dexterity results, an underdeveloped vocabulary and weak categorisation skills

- lower attention span and difficulty in judging the relevance of a document

PageFetch is a new human evaluation game that presents an IR experiment as a fun and easyto-play computer game that can be enjoyed by children and adults.
GAME STATISTICS

146 active players

85 over-16 players 57 under-16s

8672 submitted queries

1653 unique queries

1475 games completed

73 hours of games played

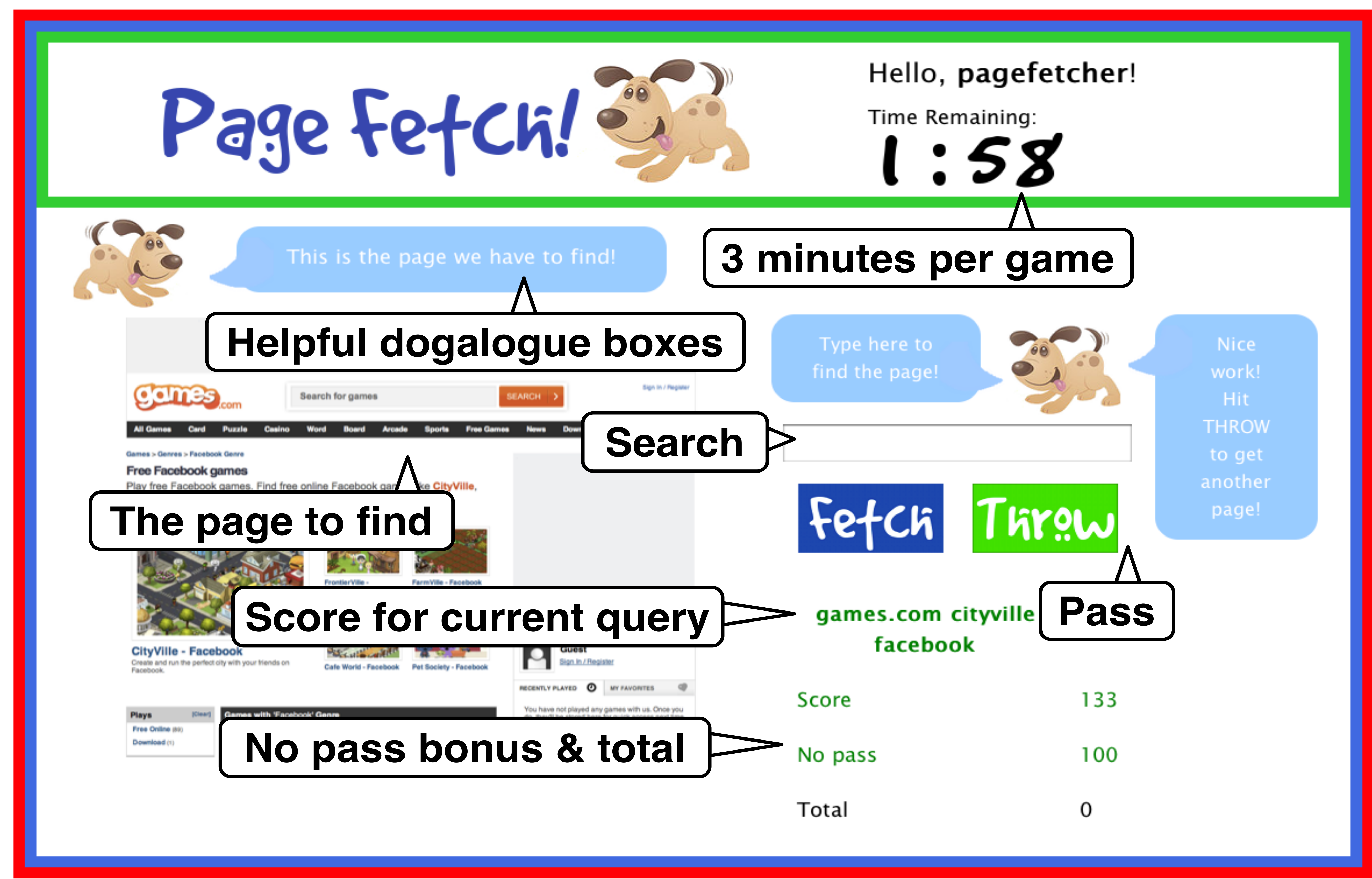

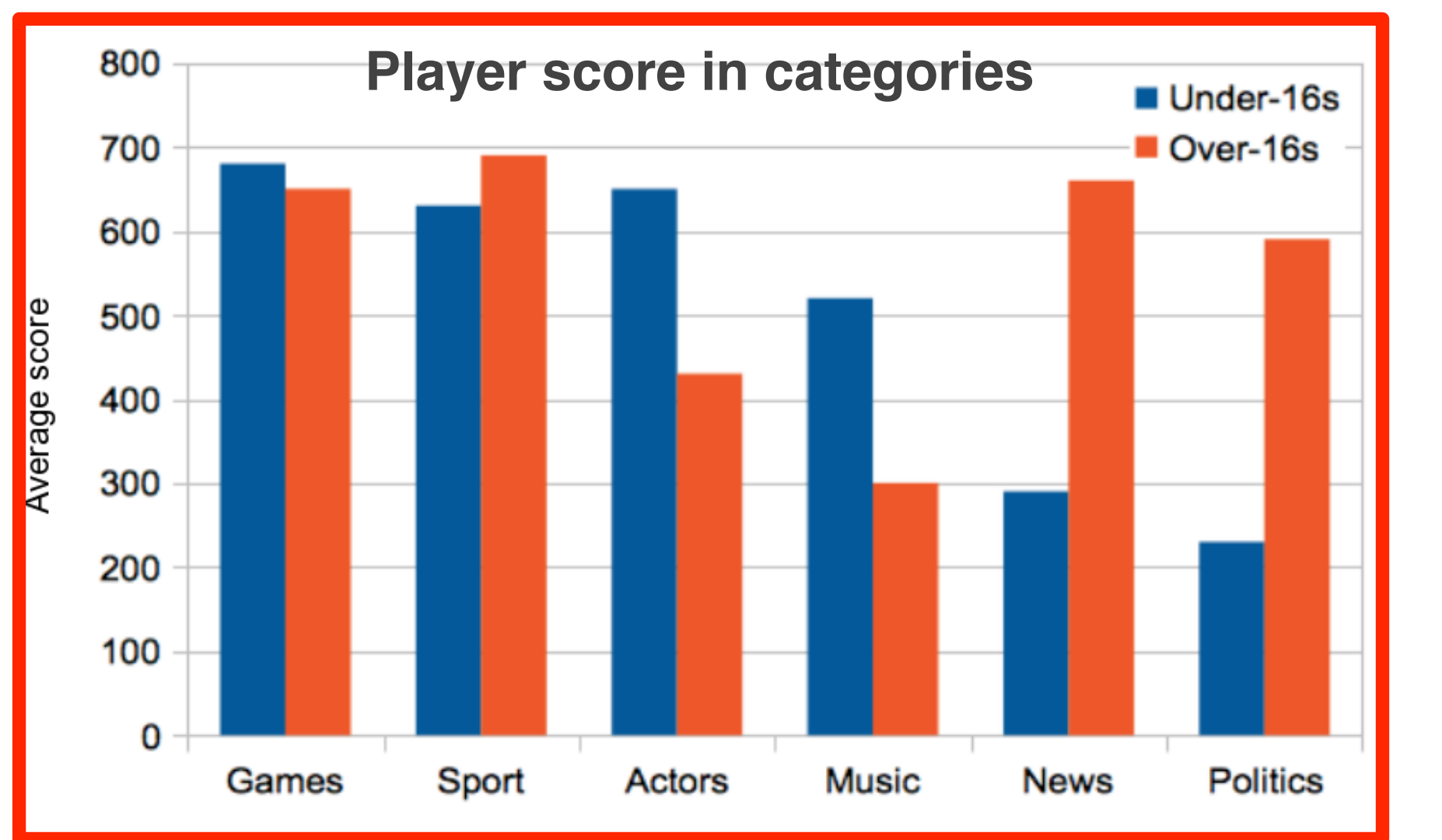

PLAY THE GAME AT:

http://www.dcs.gla.ac.uk/ access/pagefetch

\section{RESULTS AND CONCLUSIONS}

Analysis of game records and query logs revealed:

- average no pass bonus for children was 113, adults was 265.

- children scored better on child-relevant pages than pages that weren't child-relevant

- children produced fewer queries per game than adults

- children's queries were longer and had more stopwords

- children were able to give shorter, more accurate queries on child-relevant pages

- spelling didn't affect success of a query (and children misspelt queries frequently)

The use of a human evaluation game when conducting an Information Retrieval experiment proved to be an effective means of gathering large volumes of query data. Furthermore, the analysis of game results confirmed some of the assumptions made by previous IR studies. However, when searching for information that is relevant to their interests, children seem to perform better than expected, even exceeding adult searchers in some cases. 